\title{
ON PERFECT GROUP RINGS
}

\author{
S. M. WOODS ${ }^{1}$
}

ABSTRACT. It is shown that the group ring $A G$ of the group $G$ over the ring $A$ is perfect if and only if $A$ is perfect and $G$ is finite. (Perfect rings were characterized by $\mathrm{H}$. Bass in 1960.)

1. Introduction. All rings are assumed to have a unit element. A ring $R$ is called semiprime if its prime $\operatorname{radical} \operatorname{rad}(R)$ is 0 , and semiprimitive if its Jacobson $\operatorname{radical} \operatorname{Rad}(R)$ is 0 . The ring $R$ is perfect if $R / \operatorname{Rad}(R)$ is artinian and $\operatorname{Rad}(R)$ is left $T$-nilpotent (i.e. for every sequence $\left\{a_{i}\right\}$ in $\operatorname{Rad}(R)$ there exists an $n$ such that $a_{1} a_{2} \cdots a_{n}=0$ ). Equivalently, $R$ is perfect if it satisfies the descending chain condition on principal right ideals. If $R$ is a perfect ring then so is every homomorphic image of $R$, and so is $R_{(n)}$, the ring of all $n$ by $n$ matrices over $R$. These results are due to H. Bass [1].

If $A$ is a ring and $G$ is a group, $A G$ will denote the group ring of $G$ over $A$. If $H$ is a subgroup of $G$ then $\omega H$ will denote the right ideal of $A G$ generated by $\{1-h: h \in H\}$; if $H$ is normal then $\omega H$ is an ideal and $A G / \omega H \cong A(G / H)$. The fundamental ideal $\omega G$ of $A G$ will be denoted $\Delta ; A \cong A G / \Delta$. If $I$ is a right ideal of $A G$ then $I G$ will denote the right ideal of $A G$ generated by the subset $I$; if $I$ is an ideal then $I G$ is an ideal and $A G / I G \cong(A / I) G$. The group ring $A G$ is (von Neumann) regular if and only if $A$ is regular, $G$ is locally finite, and the order of every finite subgroup of $G$ is a unit in $A$; $A G$ is artinian if and only if $A$ is artinian and $G$ is finite; and $A G$ is semiprime if and only if $A$ is semiprime and $G$ has no finite normal subgroups whose orders are zero-divisors in $A$. These results may be found in Connell's paper [3] and his appendix to Lambek's book [4, Appendix 3].

This work forms part of the author's Ph.D. thesis at McGill University. Conversations with Professors J. Lambek and I. Connell were most helpful.

2. Sufficiency. In this section we assume that $A$ is perfect and $G$ is finite and show that $\operatorname{Rad}(A G)$ is left $T$-nilpotent and $A G / \operatorname{Rad}(A G)$ is artinian.

Received by the editors May 14, 1970.

AMS 1969 subject classifications. Primary 1650, 2080; Secondary 1620, 1625, 1648.

Key words and phrases. Perfect ring, group ring.

1 Preprints of some of the results of this paper have appeared under the maiden name of the author, Sheila Kaye. 
LEMMA 1. If $G$ is finite of order $n$ then there is a ring embedding of $A G$ into $A_{(n)}$ which sends $\operatorname{Rad}(A) G$ into $\operatorname{Rad}\left(A_{(n)}\right)$.

Proof. Since $A G \cong A^{n}$ as right $A$-modules, the endomorphism ring $\operatorname{End}_{A}(A G) \cong A_{(n)}$. Left multiplication by an element of $A G$ is a right $A$-homorphism of $A G$ into itself and this correspondence is clearly an embedding of the ring $A G$ in to the ring $\operatorname{End}_{A}(A G)$.

Since elements of $A$ commute with elements of $G$, an element $a$ of $A$ is mapped onto the matrix with $a$ 's on the diagonal and 0 's elsewhere. Thus $\operatorname{Rad}(A)$ is mapped into $\operatorname{Rad}(A)_{(n)}=\operatorname{Rad}\left(A_{(n)}\right)$, an ideal. The result follows.

Proposition 1. If $A$ is perfect and $G$ is finite then $A G$ is perfect.

Proof. Let $\bar{A}=A / \operatorname{Rad}(A)$. Then $\bar{A} G$ is artinian. By [3, Proposition 9], $\operatorname{Rad}(A) G \subseteq \operatorname{Rad}(A G)$. Then $\bar{A} G \cong A G / \operatorname{Rad}(A) G$ maps onto $A G / \operatorname{Rad}(A G)$ and $A G / \operatorname{Rad}(A G)$ is artinian.

The canonical epimorphism of $A G$ onto $\bar{A} G$ takes $\operatorname{Rad}(A G)$ into $\operatorname{Rad}(\bar{A} G)$, this is, $\operatorname{Rad}(A G) / \operatorname{Rad}(A) G \subseteq \operatorname{Rad}(\bar{A} G)$. Since $\bar{A} G$ is artinian, $\operatorname{Rad}(\bar{A} G)$ is nilpotent. $\operatorname{But} \operatorname{Rad}(A) G \subseteq \operatorname{Rad}\left(A_{(n)}\right)$ which is left $T$-nilpotent since $A_{(n)}$ is perfect. Thus $\operatorname{Rad}(A G)$ is left $T$-nilpotent.

\section{Necessity when $G$ is abelian.}

Lemma 2. If $A G$ is perfect then $G$ is a torsion group.

Proof. If $g \in G$ does not have finite order then the cyclic subgroups generated by $g^{2^{n}}$ for $n \geqq 0$ form an infinite descending chain. Applying $\omega$ yields an infinite descending chain of right ideals of $A G$, which are principal by $[3$, Proposition 1$]$.

Proposition 2. If $A G$ is perfect then so is $A$. If in addition, $G$ is abelian, then $G$ is finite.

Proof. If $A G$ is perfect then so is $A$ since $A \cong A G / \Delta$.

To show that $G$ is finite we may assume without loss of generality that $A=E_{(n)}$ where $E$ is a skewfield, since $A / \operatorname{Rad}(A)$ is a direct sum of rings of this type. Since $G$ is an abelian torsion group, $G$ may be written as $G_{p} \times H$, where $p$ is the characteristic of $E, G_{p}$ is a $p$-group, and the order of every element of $H$ is prime to $p$. (If $E$ has characteristic 0 take $G_{p}$ to be trivial and $H=G$.) Burgess [2] has shown that $H$ must be finite.

Suppose that $G_{p}$ is infinite. Then $A G_{p} \cong A G / \omega H$ is perfect. If $g \in G_{p}$ then $(1-g)^{p^{n}}=0$ where $p^{n}$ is the order of $g$. Since $1-g$ is in the centre, $1-g \in \operatorname{Rad}\left(A G_{p}\right)$. Construct a sequence $\left\{g_{i}\right\}$ in $G_{p}$ so 
that $g_{1} \neq 1$ and $g_{n}$ is not in the (finite) subgroup generated by $\left\{g_{1}, \cdots, g_{n-1}\right\}$. The product $\prod_{i=1}^{n}\left(1-g_{i}\right)$ is never 0 , since the term $\prod_{i=1}^{n} g_{i}$ does not cancel. This contradicts the $T$-nilpotence of $\operatorname{Rad}\left(A G_{p}\right)$.

4. Reduction to the abelian case. In this section it is shown that if $A G$ is perfect and $G$ is infinite then $G$ has an infinite abelian subgroup $H$ and $A H$ is perfect, a contradiction. Without loss of generality, we continue our assumption that $A=E_{(n)}$, where $E$ is a skewfield.

Lemma 3. If $A G$ is perfect and $H$ is a subgroup of $G$ then $A H$ is perfect.

Proof. $A G=\bigoplus_{i} A H g_{i}$ where the $g_{i}$ run over a set of coset representatives for $G / H$. If $I$ is a principal right ideal of $A H$ then $I G$ $=\oplus_{i} I g_{i}$ is a principal right ideal of $A G$. Thus a descending chain of principal right ideals in $A H$ gives rise to a similar chain in $A G$.

Lemma 4. If $I$ is a left $T$-nilpotent ideal of a ring $R$ then $I \subseteq \operatorname{rad}(R)$. Hence if $R$ is perfect, then $\operatorname{Rad}(R)=\operatorname{rad}(R)$.

Proof. The prime radical $\operatorname{rad}(R)$ is the set of all strongly nilpotent elements of $R[4, \mathrm{p} .56]$. Clearly an element of a $T$-nilpotent ideal is strongly nilpotent.

Lemma 5. A group $G$ which has infinitely many finite normal subgroups has an infinite abelian subgroup.

Proof. Without loss of generality we may assume that $G$ is the union of a countable chain of finite normal subgroups $H_{i}$. Since an infinite set of commuting elements generates an infinite abelian subgroup, if $G$ does not contain an infinite abelian subgroup then there exists a finite set $S=\left\{g_{1}, \cdots, g_{m}\right\}$ of commuting elements which cannot be enlarged. Since $G=\bigcup_{i=1}^{\infty} H_{i}, S \subseteq \bigcup_{i=1}^{n} H_{i}=H_{n}$ for some $n$. Since $H_{n}$ is finite, the index of its centraliser $C$ in $G$ is finite. Since $G$ is infinite, $C$ is infinite and so there exists $g \in C$ such that $g \notin S$. Since $g$ commutes with every element of $S, g$ may be added to $S$ and we have reached a contradiction.

Proposition 3. If $A G$ is perfect then either $G$ is finite or $G$ has an infinite abelian subgroup.

Proof. Let $A=E_{(n)}$. If $E$ has characteristic 0 then $A G$ is semiprime, hence semiprimitive by Lemma 4 . Thus $A G \cong A G / \operatorname{Rad}(A G)$ is artinian and $G$ is finite.

If $E$ has characteristic $p>0$ let $S$ be the set of natural numbers $\left\{n: G\right.$ has a normal subgroup of order $p^{n} m$ for some $\left.m\right\}$. If $S$ is finite 
let $n$ be maximal and let $H_{n}$ be a normal subgroup whose order is divisible by $p^{n}$. By the maximality of $n, G / H_{n}$ has no finite normal subgroup whose order is divisible by $p$. Therefore $A\left(G / H_{n}\right)$ is semiprime. Since $A\left(G / H_{n}\right)$ is perfect, $G / H_{n}$ is finite. Since $H_{n}$ is finite, so is $G$.

If $S$ is infinite then $G$ has infinitely many finite normal subgroups. By Lemma $5, G$ contains an infinite abelian subgroup.

This completes the proof of the theorem.

THEOREM. The group ring $A G$ is perfect if and only if $A$ is perfect and $G$ is finite.

\section{REFERENCES}

1. H. Bass, Finitistic dimension and a homological generalization of semi-primary rings, Trans. Amer. Math. Soc. 95 (1960), 466-488. MR 28 \#1212.

2. W. D. Burgess, On semi-perfect group rings, Canad. Math. Bull. 12 (1969), 645-652.

3. I. G. Connell, On the group ring, Canad. J. Math. 15 (1963), 650-685. MR 27 $\$ 3666$.

4. J. Lambek, Lectures on rings and modules, Blaisdell, Waltham, Mass., 1966. MR 34 \#5857.

University of Manitoba, Winnipeg, Manitoba, Canada 